\title{
Prospective Fathers -Their (Frequently Overlooked) Contributionto Better Reproductive Outcomes
}

\author{
Janette Roberts* \\ Diploma Clinical Nutrition, USA \\ *Corresponding author: Janette Roberts, B. Pharm (Hons), Diploma Clinical Nutrition, USA.
}

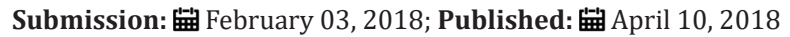

\section{Opinion}

An easy conception, healthy, full term pregnancy, short straight forward labour, long term breast feeding relationship and a child free of any metabolic, immune, neurological or digestive disorders might now be considered the exception rather than the rule with practitioners dealing more and more frequently with the many and various faces of compromised reproduction. But whether dealing with infertility, recurrent miscarriage or preventing the birth of another child with a problem, the father's role in promoting truly health reproduction is still often overlooked.

While sperm health is only one of three factors (egg \& womb being the other two) sperm are actually more vulnerable than eggs - they are smaller, more exposed and develop entirely within their present environment, with studies showing increasing contribution of the male to all conception, foetal health and pregnancy issues. Some studies indicate that as much as $75 \%$ of infertility may be due to the male, with men at least $50 \%$ responsible for the health of the baby and the pregnancy. More specifically, leukaemia, asthma, bronchial/respiratory issues and mental development/disease are all linked to the father. It has been demonstrated that there is a 10 -fold increase in testicular cancer for offspring of men exposed to organic solvents and a higher rate of respiratory disease, including asthma, particularly associated with the father smoking before conception. Miscarriage rates are closely linked to health/ morphology of sperm with a study carried out in Karolinska, Stockholm showing 14\% abnormal sperm =14\% miscarriage, $43 \%$ abnormal $=83 / 84 \%$ miscarriage. Half the miscarriages in the study were due to the male.

Sperm abnormalities have a number of causes, with environmental factors high on the list. Australian Research Council's Centre for Excellence in Bio-technology and Development is currently conducting research into effect of pollutants such as fertilizers, pesticides, cigarette smoke, medical drugs and organic solvents on declining male fertility rates, and the health of children (and grandchildren). Since men are more often in toxic occupations, professional advice must include compliance with all protective measures e.g. masks, gloves, avoidance of dust, fumes, or even move to a safer work environment.
Both toxic exposure and infection have been associated with DNA fragmentation in sperm. Not apparent in standard semen analyses, a separate test, Sperm Chromatin Structure Assay (SCSA) is required to show DNA damage. Treatment with antioxidants is of particular importance, with a significant improvement in SCSA results with intensive antioxidant therapy. TZI (Teratozoospermia Index) is a better gauge of overall health of total sperm population than percentage of perfect sperm. This index is often very low if assessed in IVF laboratories as their specialist technology can identify more subtle levels of damage. TZI gives average number of abnormalities per sperm. Aim for index $<1$.6. If index $>1.8$ there is a significant increase in miscarriage risk and lower conception rate. Other potentially detrimental lifestyle factors include exposure to heat and pressure e.g. wetsuits, saunas, spas, tight pants and bike riding (shown to be associated with prostatitis). A 2006 study published in the International Journal of Impotence showed how the "virile" image of 234 motorbike riders was often misleading with $69 \%$ of the men in the study suffering from erectile dysfunction.

Treatment for the prospective father should involve detoxifying liver herbs, methyl factors and antioxidants. Zinc, Selenium (both rich in semen to protect the sperm) CoQ10 and Lipoid Acid (also provide mitochondrial support for ageing effects and motility). In addition to reducing environmental toxicity, the prospective father's part of the bargain includes an organic, whole food diet, no alcohol caffeine or cigarettes, regular exercise, reduced stress, much reduced exposure to electromagnetic radiation (mobile phones out of pockets and laptops out of laps) and sex on a daily basis! While men may sometimes be less willing than their partners to undertake preconception healthcare, the last recommendation usually gets them excited, along with the reminder that comprehensive preconception healthcare is undoubtedly the greatest gift they will ever give their children (and their grandchildren).

Jan Roberts has spent fifty years in the health care industry. For more than thirty years she has provided information for prospective Moms and Dads. Jan is the author of six best-selling books and ebooks and three mini ebooks. She has made hundreds of presentation to professionals and prospective parents around 
the world. She believes that this important information must be widely available and engaging, when baby-making is still in the future. Both prospective parents, working together, can ensure that their future children will reach their full genetic potential! But it doesn't stop with conception-Jan is also a staunch advocate of the healthiest possible choices during pregnancy and breastfeeding, as well as the nurturing parenting practices that foster a child's emotional health, build self-esteem and promote the wellbeing of the whole family.

Read more from Jan in her books, eBook and mini ebooks (Figure 1).

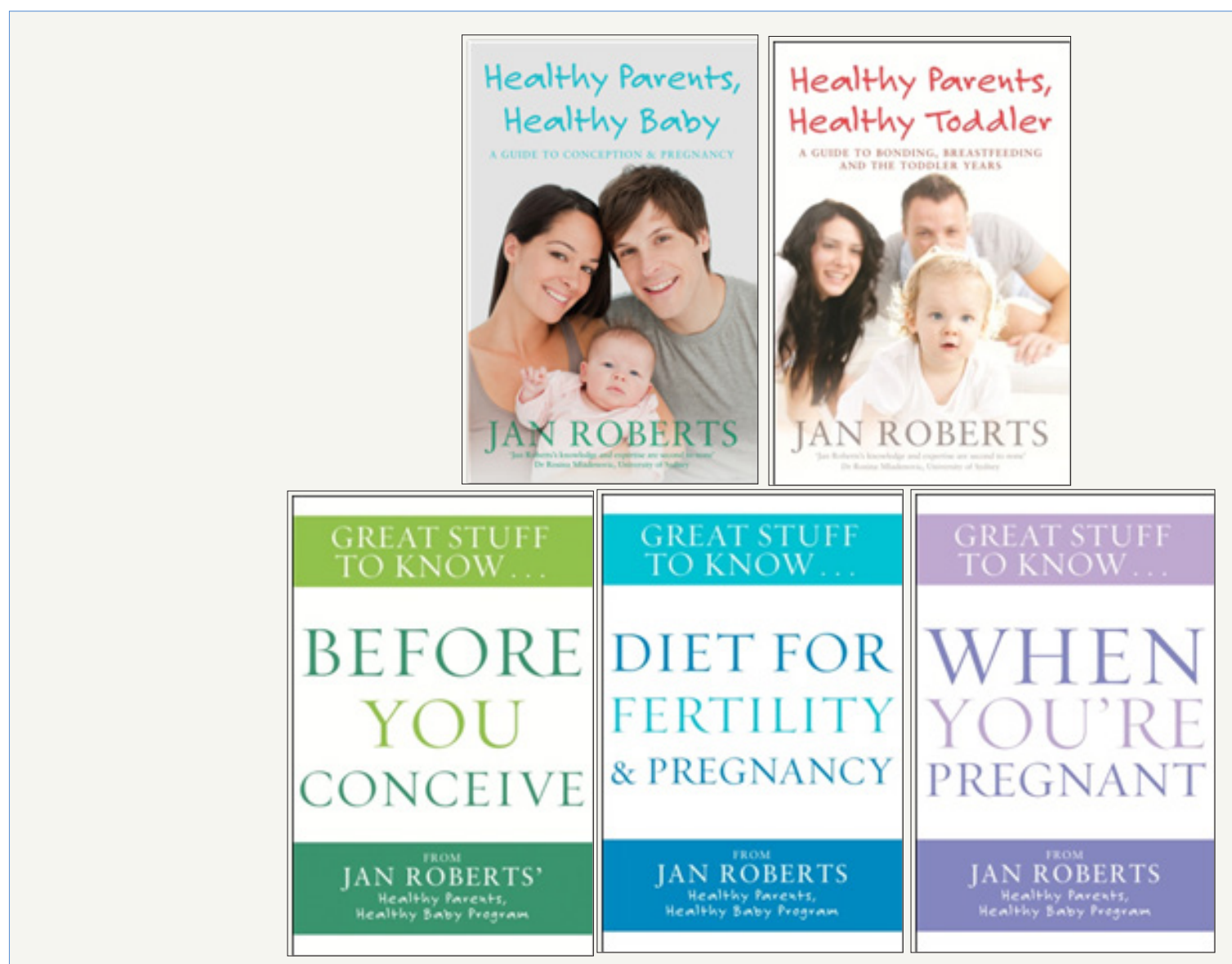

Figure 1:

Through her company Health HQ Global, Jan offers education for prospective parents, training for health professionals and consulting services to ART Clinics. To speak with Jan about including her very successful preconception program at your clinic, contact her:

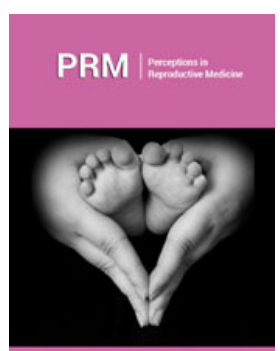

\section{Perceptions in Reproductive Medicine}

\section{Benefits of Publishing with us}

- High-level peer review and editorial services

- Freely accessible online immediately upon publication

- Authors retain the copyright to their work

- Licensing it under a Creative Commons license

- Visibility through different online platforms 